\title{
BEAM DYNAMICS SIMULATION IN A LINEAR ACCELERATOR FOR CERN FUTURE CIRCULAR COLLIDER
}

\author{
Yulia D. Kluchevskaia \\ National Research Nuclear University- \\ Moscow Engineering Physics Institute \\ Russia \\ Kluchevskaia@mail.ru
}

\author{
Sergey M. Polozov \\ National Research Nuclear University- \\ Moscow Engineering Physics Institute \\ Russia \\ SMPolozov@mephi.ru
}

Article history:

Received 29.04.2020, Accepted 14.09.2020

\begin{abstract}
Results of the beam dynamics simulation in a linear accelerator at full energy $1.5-2.0 \mathrm{GeV}$ for an international project - CERN Future Circular Collider (FCCee) are presented. FCC is developing designs for a higher performance particle collider to extend the research currently being conducted at the Large Hadron Collider, once the latter reaches the end of its lifespan. Beam dynamics simulations done using BEAMDULAC-BL code developed in NRNU MEPhI. This code allows taking into account both the quasistatic and high-frequency self-field components.
\end{abstract}

\section{Key words}

RF-gun, photogun, beam dynamics, bunch charge, bunch spectrum, longitudinal and transverse motion stability, beam loading effect, linear accelerator.

\section{Introduction}

The Future Circullar Collider (FCC) is the one of the most prospective and ambitious projects of future [Abada, 2019 EPJ C; Abada, 2019 EPJ ST 228(2); Abada, 2019 EPJ ST 228(4); Abada, 2019 EPJ ST 228(5)]. Few layouts of the electron beam injection systems were early discussed [Oide et al., 2016; Papaphilippou, 2016 FCC Meeting; Papaphilippou et al., 2016 IPAC; Ogur et al., 2018 FCC Meting; Ogur et al., 2018 eeFACT; Ogur et al., 2019]. But all layouts will include the e-linac with energy of few GeV. The beam dynamics simulations were done at NRNU MEPhI [Bondarenko et al., 2016; Bondarenko et al., 2017] using BEAMDULAC-BL code developed at MEPhI [Masunov et al., 2006; Masunov et al., 2008; Masunov et al., 2010; Bondarenko et al., 2013; Polozov, 2018]. As it was discussed many times, we have a possibility to use the scheme of injector with two types of the first sections (1) RF-gun with photocathode for $300 \mathrm{pC}$ bunches for injection into booster (RF-gun-v2); (2) RF-gun with thermocathode as an option for high intensity $6 \mathrm{nC}$ drive bunches production for e-/e+ conversion (RF-gun-v3). The possible layout of linac is presented in Figure 1. The beam dynamics simulation was presented and discussed in [Bondarenko et al., 2016; Bondarenko et al., 2017]. The main results are described below:

I) A $300 \mathrm{pC}$ and $10 \mathrm{ps}$ bunch can be easily accelerated in RF-gun-v2 version (results of beam dynamics simulation in RF-gun-v1 are presented in [Bondarenko et al., 2016]). Here and following all simulations done for $3000 \mathrm{MHz}$ structures. The current transmission coefficient is close to $100 \%$ and RF field amplitude of $600 \mathrm{kV} / \mathrm{cm}$ is quite enough to have $10.5 \mathrm{MeV}$ after photogun. Such energy is necessary for effective recapturing by the second section, as it was presented at FCC Meeting 2016. The bunch energy spread FWHM (full width at half maximum) is $\pm 3 \%$ (or $\pm 300 \mathrm{keV}$ ) and we can suppose that output energy spread after 10 or 20 regular sections with $\beta_{\mathrm{ph}}=1$ will not be higher than 0.5$1.0 \%$. Beam loading effect is not sufficient here: one bunch decreases RF field amplitude less than $0.5 \%$ and such beam loading can be easily compensated by RF feed system. Transverse focusing can be effective using solenoid of $0.1 \mathrm{~T}$ on axis.

II) Beam dynamics in RF-gun-v2 structure was also simulated for 1-6 $\mathrm{nC}$ bunches. It was shown that the current transmission coefficient will drop fast for high bunch charge, as example it will not be higher than $60 \%$ for $6 \mathrm{nC}$ bunches. The RF-gun-v2 will have current limitation due to this and cannot be effectively used as $\mathrm{nC}$ bunch accelerator for bunches 


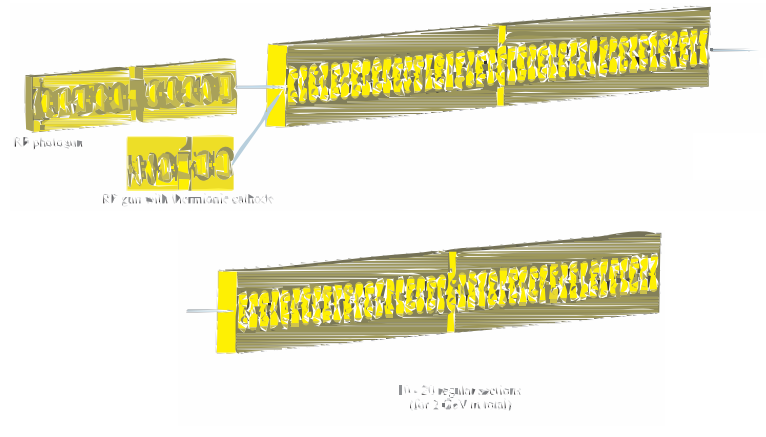

Figure 1. Two possible schemes of linac layout (RF gun with thermionic cathode is).

with population higher of $2 \mathrm{nC}$. The bunch spectrum width will also increase fast vs the bunch charge.

III) Simulations show that high bunch intensities lead to high back currents. Sufficient Coulomb "headtail" repulsion was observed. Head-tail difference of RF field amplitude due to high bunch phase size and beam loading effect leads to energy spectrum growth.

IV) One interesting effect was observed for high intensity bunches: beam spectrum and capturing coefficient are sufficiently dependent on initial bunch phase distribution. Two phase distributions were used: Kapchinsky-Vladimirsky (KV) and uniform. The capturing coefficient is $\sim 5 \%$ higher for $\mathrm{KV}$ and energy spread is less for KV distribution. It can be possible that for short (100 fs-1 ps) laser pulses a phase distribution is close to uniform, but for higher pulse durations it can be different.

V) We need to do more intensive studies of nearcathode processes including review of references in field of photo emission. Bunch duration of $10 \mathrm{ps}$ is much higher than relaxation time in metal and we can have an electron depletion for laser exposed volume. Back current influence the beam emission, double layer problems and emission processes including possible depletion should be studied in details.

VI) RF-gun with thermocathode (RF-gun-v3) was proposed to accelerate $\mathrm{nC}$ bunches. Beam dynamics simulations results shows that for such gun the capturing coefficient can be increased to $90-95 \%$ for $3 \mathrm{nC}$ bunches and to 70-80\% for $6 \mathrm{nC}$ bunches. But FWHM energy spectrum enlarges to $\pm 20 \%$ for $6 \mathrm{nC}$ bunches compared to $\pm(8-11) \%$ for photogun_v2. But here we should note, that energy spectrum for thermionic guns is defined not only by Coulomb repulsion but by phase velocities and RF field amplitudes in first 2-4 cells where electron velocities are sufficiently less than 1 . Energy spread can be decreased by initial cells parameters optimization (it is planned for future simulations). The optimal injection energy for RF-gun with thermocathode is $100 \mathrm{keV}$.

\section{RF-gun with Thermionic Cathode and Beam Dy- namics}

As it is clear we have no problems for $300 \mathrm{pC}$ bunches generation and acceleration but for $6 \mathrm{nC}$ bunches the structure and beam dynamics simulation results will be sufficiently improved.

First initial cells of the RF-gun with thermionic cathode were optimized to increase the capturing coefficient. The optimized version (called RF-gun-v4) will have three cells with adiabatically increasing phase velocity $\beta_{\mathrm{ph}}=0.92,0.96,0.99$ (compared to four cells for $R F$ - gun-v3 with $\left.\beta_{\mathrm{ph}}=0.9,0.91,0.98,0.99\right)$. The solenoid field also should be increased up to $0.6 \mathrm{~T}$ on the channel axis ( $0.4 \mathrm{~T}$ for $R F$-gun-v3) to control the beam envelope and transverse emittance.

The RF-gun-v 4 structure and the bunch have the following characteristics: the structure consists of 8 accelerating and 7 coupling cells, there are no side coupled cells, first three cells have the phase velocities less than 1, last accelerating cell have no coupling one and its length was enlarged, total section length is $\sim 31 \mathrm{~cm}$, channel aperture radius $10 \mathrm{~mm}$, coupling cell length is $4 \mathrm{~mm}$, diaphragm thickness $4 \mathrm{~mm}$, shunt impedance $\sim 80 \mathrm{MOhm} / \mathrm{m}$. The simulation was done for bunch charge $6 \mathrm{nC}$ and bunch duration of $10 \mathrm{ps}$, the injection energies was chosen equal to $50 \pm 0.5 \mathrm{keV}$, $100 \pm 1.0 \mathrm{keV}, 200 \pm 2.0 \mathrm{keV}$, the initial transverse emittance is $20 \mathrm{~mm} \cdot \mathrm{mrad}$. The results of the beam dynamics simulations are given in Figures 2-5 for RF fields of $800 \mathrm{kV} / \mathrm{cm}$ and $1000 \mathrm{kV} / \mathrm{cm}$.

Beam dynamics simulation results shows that the capturing coefficient is $\sim 85-90 \%$ for $6 \mathrm{nC}$ bunch (comparatively 70-80\% for RF-gun-v3) depending on injection phase, initial phase distribution and bunch current, output energy about 6-7 MeV for $800 \mathrm{kV} / \mathrm{cm}$ and $1000 \mathrm{kV} / \mathrm{cm}$, output beam spectrum FWHM $\pm 20-40 \%$. The main remarks can be done after next simulations:

(1) Beam dynamics, including capturing, Coulomb repulsion, head-tail effects, energy spread are defined by the bunch charge and by the initial phase-energy distribution;

(2) as it can be seen in Figures 3-5, KapchinskyVladimirsky (KV) initial phase distribution give better results compared to normal one;

(3) the field distribution in the first accelerating cells play the key role in bunch formation and, further, to capturing coefficient and energy spread;

(4) it should be noted that $1000 \mathrm{kV} / \mathrm{cm}$ give no preferences for beam dynamics compared to $800 \mathrm{kV} / \mathrm{cm}$;

\section{Photogun Beam Dynamics}

The RF-gun-v2 structure also was optimized for decrease the energy spread and to enlarge the capturing coefficient. After that the RF-gun-v2 structure and 
the bunch have the following characteristics: the structure consists of 8 accelerating and 7 coupling cells, there are no side coupled cells, first three cells have the phase velocities of $\beta_{\mathrm{ph}}=0.92$ (half cell), 0.96, 0.99, last accelerating cell have no coupling ones and its length was enlarged, total section length is $\sim 31 \mathrm{~cm}$, channel aperture radius $10 \mathrm{~mm}$, coupling cell length is $4 \mathrm{~mm}$, diaphragm thickness $4 \mathrm{~mm}$, shunt impedance $\sim 80 \mathrm{MOhm} / \mathrm{m}$, solenoid field was varied from 0.05 to $0.6 \mathrm{~T}$.
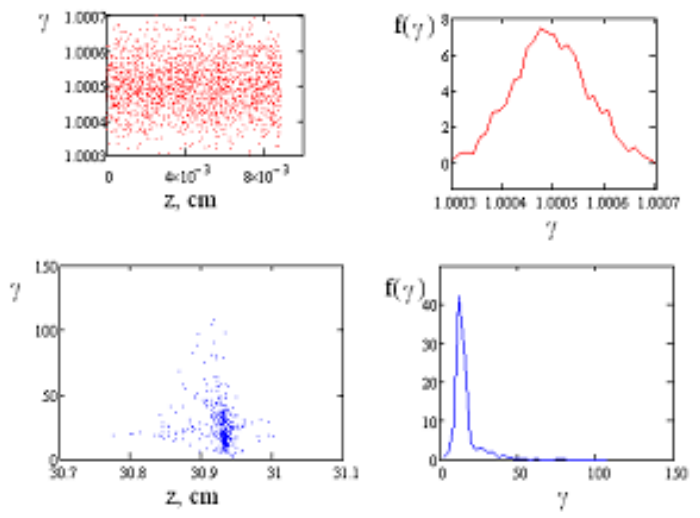

Figure 2. Phase portraits and output energy spectrums for different bunch injection phase, injection energy $100 \mathrm{keV}, E_{z}=800 \mathrm{kV} / \mathrm{cm}$, the bunch charge $6 \mathrm{nC}$, uniformly and $\mathrm{KV}$ initial phase distribution.

The simulation was done for bunch charge $6 \mathrm{nC}$ and bunch duration of $10 \mathrm{ps}$, the initial transverse emittance is $20 \mathrm{~mm} \cdot \mathrm{mrad}$. Some initial phase-energy particles distributions were simulated: normal and KV phase distribution were studied, the initial energy spread influence was also discussed. The results of the beam dynamics simulations are given in Figure 5 for RF fields of $E_{z}=800 \mathrm{kV} / \mathrm{cm}$.

It is clear that RF-gun-v3 can realize more effective bunch capturing compared to RF-gun-v2 version, but electron's losses of $15-20 \%$ are still very high. Higher solenoid focusing field give us not only higher capturing coefficient but also the lower energy spread. It should be noted that the phase loses for all simulation variants are close to $15-17 \%$, other particles are lost transversally. For all variants the optimal injection phase $\delta \phi$ is close to 3.4. In case when $\delta \phi=3.0-3.2$ the higher energy $\approx 12$ $12.5 \mathrm{MeV}$ is observed, but the energy spectrum FWHM is wider and equals $\approx \pm 15-19 \%$, current transmission coefficient is the same. For the injection phase $\delta \phi=3.5$ the energy spectrum is much better $\approx \pm 7-8 \%$, but output energy is $\approx 5-5.5 \mathrm{MeV}$.

All results noted above leads to an evident conclusion: beam dynamics of the high-intensity bunch, capturing efficiency, energy spread are defined by the bunch emission process, RF and magnetic field values near cathode and Coulomb effects at the first $2-3 \mathrm{~mm}$ of trajectory where electrons are non-relativistic and ultra-relativistic.

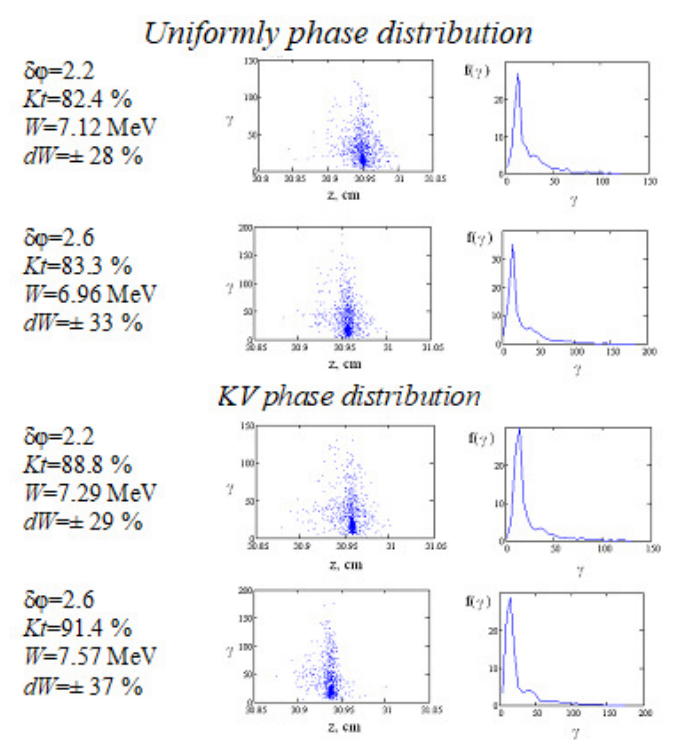

Figure 3. Phase portraits and output energy spectrums for different bunch injection phase, injection energy $50 \mathrm{keV}, E_{z}=800 \mathrm{kV} / \mathrm{cm}$, the bunch charge $6 \mathrm{nC}$, uniformly and $\mathrm{KV}$ initial phase distribution.

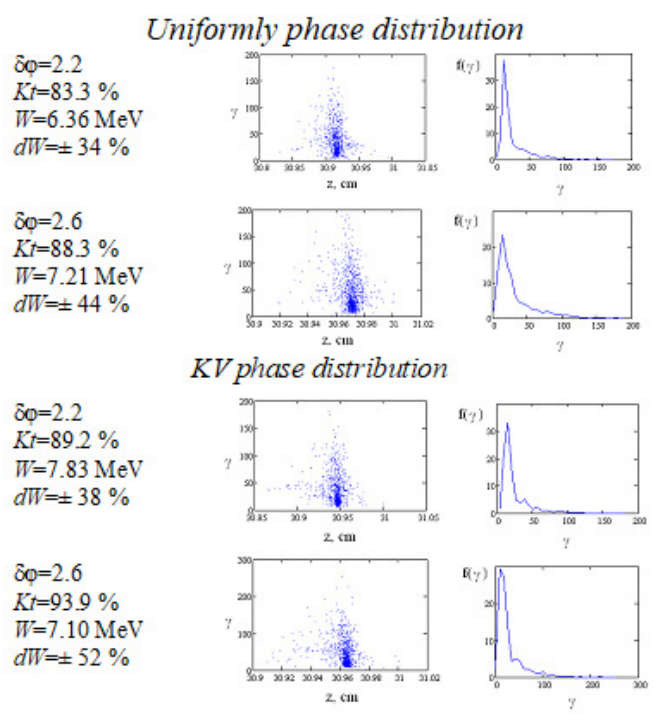

Figure 4. Phase portraits and output energy spectrums for different bunch injection phase, injection energy $50 \mathrm{keV}, E_{z}=1000 \mathrm{kV} / \mathrm{cm}$, the bunch charge $6 \mathrm{nC}$, uniformly and $\mathrm{KV}$ initial phase distribution.

The electrons loses of 15-20\% are much lower than for RF-gun-v2 version ( $\sim 40 \%$ ) but they should be sufficiently neglected because about half of the non-captured electrons forms the back-current. We cannot propose a 


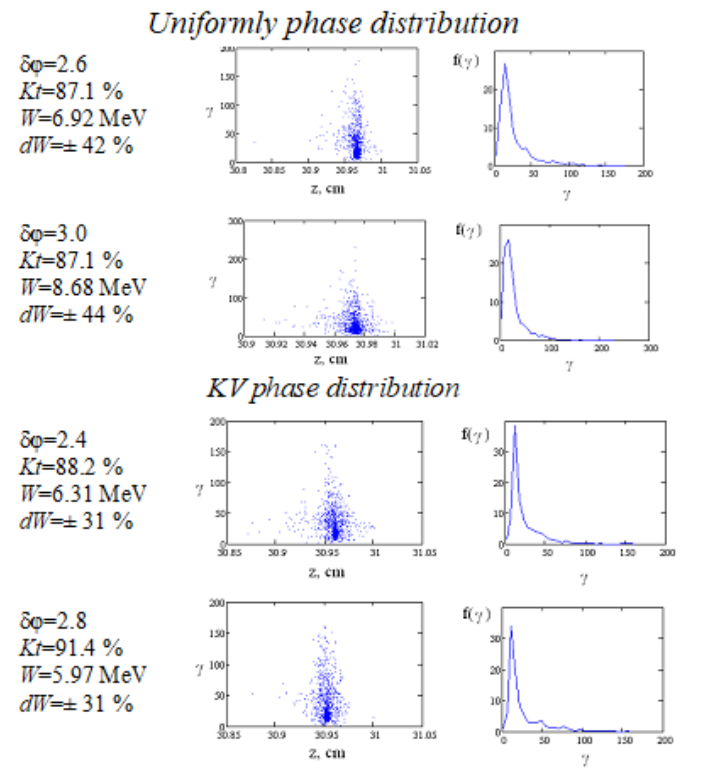

Figure 5. Phase portraits and output energy spectrums for different bunch injection phase, injection energy $100 \mathrm{keV}, E_{z}=800 \mathrm{kV} / \mathrm{cm}$, the bunch charge $6 \mathrm{nC}$, uniformly and $\mathrm{KV}$ initial phase distribution.

priory as the back-current of $600 \mathrm{pC}$ will influence the electrons emission but it is clear that it will have a negative influence to the cathode life-time.

Beam dynamics simulation results depending also on electric field amplitude distribution in photogun cells are presented in the Figure 6. During the simulation, we considered both the forms of the field amplitude typical of structures with $\pi$ mode (Figure $6 \mathrm{a}-\mathrm{f}, \mathrm{i}$ ), as well as more exotic ones that are usually not found in practice (Figure $6 \mathrm{~g}$ and $\mathrm{h}$ ).

Beam dynamics simulation results using form of RF field amplitude in the regular cell shown on Figure 6(h) are presented below. The current transmission coefficient is also close to $100 \%$ but RF field amplitude of 600 $\mathrm{kV} / \mathrm{cm}$ is enough to have only $5.7 \mathrm{MeV}$ after photogun. Such energy may be insufficient for effective recapturing by the second section. The bunch energy spread FWHM is $\pm 8 \%$ (or $\pm 800 \mathrm{keV}$ ) and after several regular sections will be higher than $10 \%$, that is also not acceptable result. Beam cross section, phase portrait and output energy spectrum are shown in Figure 7. This result proves one more time that the RF field distribution should be controlled not only for the half-cell of the photogun but for regular cells also.

\section{Conclusions}

Results of beam dynamics simulation and electrodynamics study for the new FCC-ee injector linac were presented. Two possible schemes of linac layout were discussed: with single RF photogun, with RF photogun for injecting beam into the booster and RF-gun with thermocathode to drive beam for the e-/e+ conversion. It was shown that photogun can produce high-quality bunches of hundreds $\mathrm{pC}$ for injection as one could expect. But the capturing coefficient falls for high bunch charge and the bunch quality sufficiently decreases.

A RF-gun with the thermocathode is free from these limitations and can be used to produce drive bunches with high efficiency. But energy spectrum FWHM enlarges to $\sim \pm 20 \%$ for $6 \mathrm{nC}$ bunches in thermogun compared to $\pm(8-11) \%$ for RF-gun-v2. Beam loading influence is not sufficient for hundreds $\mathrm{pC}$ bunches but should be compensated for drive $\mathrm{nC}$ bunches.
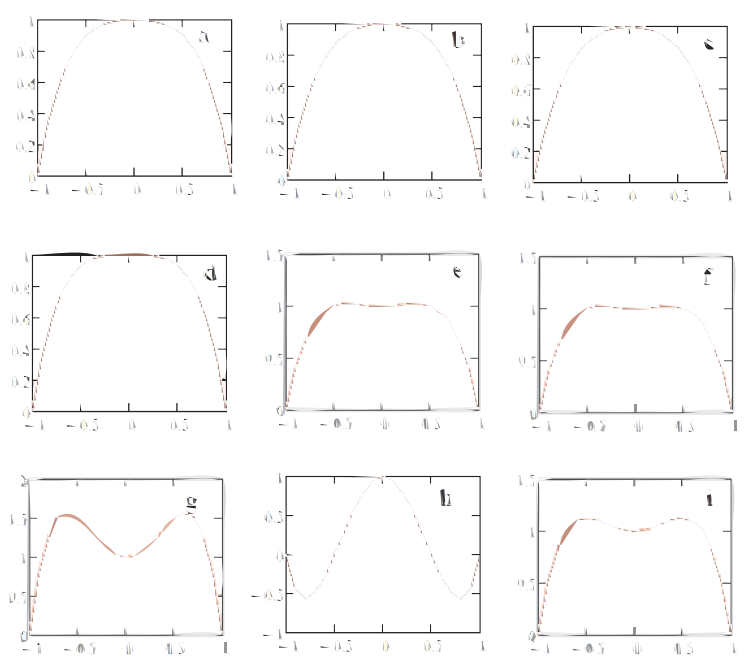

Figure 6. Variants of electric field amplitude distribution on cell's axis.
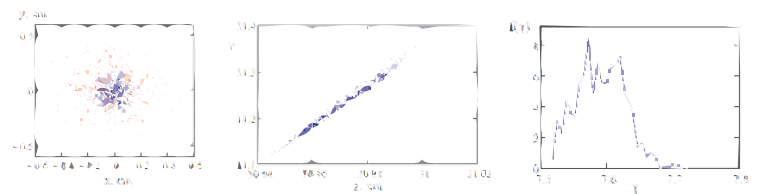

Figure 7. Beam cross section (the initial distribution is shown in red, the output in blue), phase portrait and output energy spectrum, injection energy $50 \mathrm{keV}, E_{z}=600 \mathrm{kV} / \mathrm{cm}$, the bunch charge $6 \mathrm{nC}$.

A conventional biperiodical acceleration structure (BAS) with the magnetic coupling windows was studied as the base accelerating structure. The high coupling coefficient of 10-12\% can be met with BAS. It leads to low time of the transient process and SLED (Stanford Linac Energy Doubler) or any other RF power pulse compression technique can be used for new linac. Any beam loading compensation method should be used for drive bunches acceleration, but loading is much lower for injecting bunches. 


\section{Acknowledgements}

The reported study was funded by RFBR, project number 19-29-12036.

\section{References}

Abada, A. et al. (2019). FCC Physics Opportunities Future Circular Collider Conceptual Design Report Volume 1. European Physical Journal, 79(6), p. 474.

Abada, A. et al. (2019). FCC-ee: The Lepton Collider - Future Circular Collider Conceptual Design Report Volume 2. The European Physical Journal Special Topics, 228(2), pp. 261-623.

Abada, A. et al. (2019). FCC-hh: The Hadron Collider - Future Circular Collider Conceptual Design Report Volume 3. The European Physical Journal Special Topics, 228(4), pp. 755-1107.

Abada, A. et al. (2019). HE-LHC: The High-Energy Large Hadron Collider - Future Circular Collider Conceptual Design Report Volume 4. The European Physical Journal Special Topics, 228(5), pp. 11091382.

Bondarenko, T.V. and Polozov, S.M. (2016). First Results of Beam Dynamics Simulation in electron injector linac for FCC-ee. In Proc. of RuPAC-2016, TUPSA025, p. 264-266.

Bondarenko, T.V. and Polozov, S.M. (2017). Beam Dynamics Simulation in Two Versions of New Photogun for FCC-ee Electron Injector Linac. In Proc. of IPAC'2017, TUPAB011, pp. 1326-1329.

Bondarenko, T.V., Masunov, E.S., and Polozov, S.M. (2013). Package of programs BEAMDULAC-BL for 3-D electron beam dynamics simulation taking into account own electrostatic field and current loading. Problems of Atomic Science and Technology Nuclear Physics Investigations 6 (88) p. 114-118.

Masunov, E.S. and Polozov, S.M. (2006). BEAMDULAC code for numerical simulation of 3D beam dynamics in a high-intensity undulator linac. Nuclear Instruments and Methods in Physics Research, 558, pp. 184-187.

Masunov, E.S. and Polozov, S.M. (2008). The new version of BEAMDULAC code for high intensity ion beam dynamics. Phys. Rev. ST AB, 11, 074201.

Masunov E.S., Polozov S.M., Rashchikov V.I., and Voronkov, A.V. (2010). Calculation of beam dynamics in traveling-wave accelerators taking account of the current load. Atomic Energy, 109,(2), pp. 106-112.

Oide, K. et al. (2016). FCC-ee Machine Layout and Beam Optics, FCC Meeting, Rome, Apr. 11-15.

Ogur, S. et al. (2018). Layout and Performance of the FCC-ee Pre-Injector Chain. J. Phys.: Conf. 1067, 022011.

Ogur, S. et al. (2018). Overall Injection Strategy for FCC-ee. In Proc. of eeFACT'2018, pp. 131-138.

Ogur, S. et al. (2019). Linac and Damping Ring Designs for the FCC-ee. In Proc. of IPAC'2019, pp. 420-423.

Papaphilippou, Y. (2016). FCC-ee injector complex including Booster, FCC Meeting 2016, Rome, Apr. 1115 .

Papaphilippou, Y. et al. (2016). Design Guidelines for the Injector Complex of the FCC-ee. In Proc. of the IPAC'16, pp. 3488-3490.

Polozov, S.M. (2018). BEAMDULAC-multibunch-tw. Certificate for the code registration RU 2018661944. 